\title{
BREEDING FOR DISEASE RESISTANCE IN FOREST TREES ${ }^{1}$ \\ BY C. HEIMBURGER ${ }^{2}$ \\ ABSTRACT
}

Breeding for disease resistance in forest trees is a specialized kind of forest tree breeding. With breeding of white pines for resistance to blister rust as an example, the various problems encountered and solved are described. Resistance to blister rust in eastern white pine has thus far been found to be inherited on a polygenic basis. This influences the choice of effective breeding methods and the silvicultural use of the resistant materials obtained. The genetic basis of superior resistance found in exotic species, such as Balkan white pine, Japanese white pine and Himalayan white pine is also influencing the breeding methods. Because of its early flowering, breeding work with Balkan white pine has progressed further than with other exotic species. Indications have been obtained that resistance in this species is also based on polygenes. Some of these are complementary to those found in eastern white pine. In Himalayan white pine materials the presence of recessive major genes for resistance as well as polygenes is probable. The possible use of these findings in the development of resistant white pine materials and their use in the establishment of artificially and naturally regenerated stands is discussed.

INTRODUCTION

Breeding for disease resistance is a very wide topic. This presentation will deal specifically only with certain aspects of breeding for disease resistance in forest trees, meaning disease caused by parasitic fungi. Very little has as yet been done in this field generally; therefore this paper will draw rather heavily on our own work at Maple, Ont. with breeding of white pines (Pinus L., subgen. Haploxylon Koehne) for resistance to blister rust (Cronartium ribicola Fischer). The results may not all be suitable for generalization but should contribute to a better understanding of the problems and procedures involved in this particular field.

Diseases are, of course, also caused by ecological or environmental factors affecting the health of forest trees, such as, for instance, the effect of extreme weather conditions, of smelter fumes and so on, and will not be dealt with at present. Thus by disease in the above narrower sense is meant an abnormal condition in a tree caused mainly by a fungus attack, and its manifestation is also influenced to a varying degree by the environment. Breeding involves manipulation of the genotype and thus has a direct bearing on the genotypic component of the reaction of forest trees to fungus attacks.

Breeding for disease resistance in forest trees is encouraged by the promising results with breeding for resistance in agricultural and horticultural crops; the introduction of parasitic fungi (and insects) from other continents to North America; the very serious economic losses following such intro-

\footnotetext{
${ }^{1}$ Paper presented to the Central International Forest Insect and Disease Conference, Sault Ste Marie, Ontario, September 14, 1961. Contribution No. 62-4, Research Branch, Ontario Department of Lands and Forests, Maple, Ont.

${ }^{2}$ Biography in For. Chron. $31(1): 60,1955$.
} 
ductions; the very certain possibilities of increased introduction of foreign pests in the present jet age, with resulting economic losses.

Most forest tree breeding activities are concerned with so-called forest tree improvement. This is mainly a selection process based on presumed good correlations between phenotypic expression and genetic basis of several economically important forest tree characteristics, involving mainly growth form, wood quality and the numerous factors influencing yield. Breeding in the more strict sense includes a choice among several available procedures leading towards the development of new kinds of forest trees with the most economical expenditure of time, space and other nursery and plantation efforts. It is also to a very high degree based on the genetic situation governing the expression of the desired quality in the tree materials used. Breeding for resistance to disease caused by parasitic fungi in forest trees is merely a more specialized kind of forest tree breeding, based on the genetic situation governing host-parasite interaction, causing the phenotypic expression of a disease on the background of any given set of environmental factors.

$\therefore$ ind

\section{Degrees of Fungus AtTack}

The degree of a fungus attack depends on the genetic background of the host, governing resistance on the one hand and the response of the host to any given set of environmental factors on the other. It is also influenced by the genetic background of the parasite concerned, governing virulence and response to environment (5). Thus in white pines, resistance to blister rust is governed by the genetic backgrounds of the pine materials and the blister rust at hand, and by the genetic backgrounds of the response of the pine and the blister rust to the environment. As blister rust is a heteroecious fungus, the degree of attack on pine is influenced by the degree of attack on Ribes, the alternate host, which again is governed by the two main kinds of genetic background of the Ribes.

The degree of attack is also governed by the kind of parasitism and by the environment. Since degree of attack by obligate parasites is usually positively correlated with the vigour of the host, it is important to grow host materials under as favourable conditions as possible when breeding for resistance to a disease caused by an obligate parasite. This means, not only growing the test plants in suitable soil but also satisfying their other environmental requirements, so that they grow well at the time of artificial inoculation and natural infection.

In the case of facultative parasites, the degree of an attack is often negatively correlated with the vigour of the host concerned. This should not mean that we should intentionally starve forest trees to be selected for resistance. Rather, selection should be directed towards individuals showing better than average growth rate on average sites for the species concerned. Any expression of heterosis (6), resulting from more efficient than average nutrient uptake, assimilation and adaptation to site would then be of selective value in resistance breeding. By far the most forest plantation diseases are caused by facultative parasites, attacking trees on sites to which the trees are inadequately adapted. In most cases better attention to site and other 
silvicultural factors, at time of establishment and during maintenance, will solve this problem. In some cases it is of economic advantage to grow certain tree species on sites where they do not occur under natural conditions and to which they may be rather poorly adapted to begin with. They are then often subjected to diseases caused by a combination of poor adaptation to site and attack by facultative parasites. In this case superior adaptation to such a new site would be a factor to be considered in breeding, together with resistance to the disease in question.

The genetic background of resistance in the host can be polygenic or be governed by a smaller number of major genes, although in most cases resistance to disease in plants has been found to be based on a combination of polygenes and major genes. Major genes governing resistance to disease constitute the basis for most of the spectacular advances in breeding for disease resistance in agriculture and horticulture (1).

\section{Resistance Based on Polygenic Inheritance}

Breeding for resistance on the background of polygenic inheritance in the host usually involves a search for very rare resistant individuals. The result of screening is then at first a rather slow rise in the frequency of resistant individuals in the succeeding generations, followed by more rapid advances, until a plateau is reached beyond which further advances are insignificant. This plateau may or may not be sufficient for an economic degree of resistance. Further advances in breeding are possible by inclusion of additional populations of the host showing some resistance. Hybridization of resistant individuals from two such populations may then yield mostly susceptible progenies because of complementary action of the genes governing susceptibility. Further advances in resistance may appear only in the F2 and succeeding generations. Extensive progeny tests, to determine heritability of resistance and combining ability of certain resistant individuals, are necessary to bring about relatively rapid advances in this rather slow and laborious process.

It must be kept in mind, however, that most cultivated plants have been produced by selection and breeding mostly on the basis of polygenes and that many new and useful characteristics, such as disease resistance can be materially enhanced in this manner if no other closely related materials with superior resistance are available. It took several hundreds, up to thousands, of years to develop some of our cultivated plants from their wild ancestral forms but it need not take nearly that long with our present background of genetics and breeding methods derived from it.

Our work with eastern white pine, Pinus strobus L., is a good example of breeding for resistance on a background of polygenic inheritance of resistance. Resistance in unselected white pine materials has thus far been found to be very low. After screening (infection under especially favourable conditions to the rust) the remaining materials exhibit a somewhat higher degree of resistance and this resistance seems to be inherited in a polygenic manner. Openpollinated offspring of resistant trees are almost as susceptible as unselected materials, while full-sib progenies of resistant trees show a varying degree of increase of resistance, depending on the breeding value (kind and number 
of polygenes) of the parents. The genes for resistance are very rare in all populations sampled thus far and thus may be slightly deleterious or linked with deleterious genes under natural conditions, for otherwise they would not be so rare. Because of simple mass action inherent in most polygenic systems, resistance at first appears to be recessive in crosses with unselected materials. Only a slow accumulation of genes governing resistance should gradually increase the proportion of resistant seedlings in successive generations obtained from crossing of resistant parents. The increase will probably be rather slow at first, then rise as an increasing number of seedlings become homozygous in respect to resistance genes. Sooner or later a plateau is expected to be reached, depending on the gene pool of the materials at hand. If the plateau still is below the economic level of resistance (a minimum of $30 \%$ resistant seedlings) other populations, preferably from more distant geographic regions, will have to be included in the breeding program. In order to obtain as large a gene pool in this respect as possible, it is advisable to include as wide a population sample of the species as the climatic and soil conditions of the place of testing allow, and also to work on the interspecific level. Several parallel breeding procedures are thus possible and desirable.

The situation in western white pine ( $P$. monticola Dougl.) appears to be less polygenic than in $P$. strobus, i.e. there is probably a smaller number of genes involved governing resistance, but each with a somewhat stronger expression in the phenotype than in $P$. strobus. This is also indicated by the more pronounced difference between resistant and susceptible seedlings of this species in screening for resistance as compared with seedlings of $P$. Strobus.

The situation in the highly resistant Balkan white pine ( $P$. peuce Griseb.) appears to differ from the above in that hybrids with $P$. strobus are largely susceptible to blister rust. However, crosses with some of our best (yielding resistant seedlings) combining $P$. strobus have thus far yielded much larger proportions of resistant seedlings than crosses of unselected $P$. strobus with $P$. peuce. The latter can thus be assumed to carry a number of resistance polygenes with an expression that is additive to those of $P$. strobus. Our results are in this respect similar to the results of inter-crossing selected resistant $P$. monticola and $P$. strobus reported elsewhere (2).

The few crosses of the highly resistant Japanese white pine ( $P$. parviflora Sieb \& Zucc.) with $P$. strobus made thus far have yielded largely susceptible seedlings. The situation in this species may thus resemble that found in $P$. peuce.

One useful character of $P$. peuce is precocious flowering which can be used as a very important tool in breeding. Precocious types can be found in birch, chestnut, larch, pines, poplar and often appear after interspecific hybridization indicating combined action of complementary genes influencing age at first flowering. Such genes are, of course, injurious to direct timber production and will have to be eliminated in later generations after they have served their purpose in making possible relatively rapid advances in breeding work with short breeding cycles during the initial slow stages of a breeding program.

The situation in the usually highly resistant Himalayan white pine 
( $P$. griffithii McClelland) is somewhat similar to that found in $P$. peuce and $P$. parviflora. In some crosses with unselected $P$. strobus fairly high proportions of resistant seedlings were obtained while other crosses yielded largely susceptible seedlings. This species has not been found as resistant to blister rust in our tests as $P$. peuce and $P$. parviflora in that some clonal lines and seedling populations showed fairly high proportions of infected individuals after screening for resistance. Some crosses with selected $P$. strobus have yielded very high proportions of seedlings free from blister rust after one screening, but such results cannot as yet be considered definite. This species occupies a much wider geographical and altitudinal range than $P$. peuce and $P$. parviflora, and has shown a rather wide variety of adaptation to the growing conditions of Maple, Ont. depending on provenance. It is probable that rather strong differentiation into geographic and altitudinal races is obtaining; this may include intraspecific differences in the genetic basis for resistance to blister rust.

\section{Resistance Based on Major Genes}

Breeding for resistance on the background of inheritance based on major genes is the most commonly employed method in agriculture and horticulture if such genes can be found in the host species, or can be introduced into it from other, related species. If the expression of the genes is dominant in hybrids with susceptible materials, the simple method of backcrossing with concurrent testing, to maintain resistance, is employed. Even if the major genes governing resistance are recessive in the hybrids, relatively rapid advances are possible that yield rather spectacular results, at least for the time being.

We have possibly a case of major genes influencing resistance to blister rust in $P$. griffithii. Three such pines growing in Rochester, N.Y., yielded largely resistant seedlings when intercrossed. In crosses with unselected $P$. strobus most of the seedlings were susceptible to blister rust. Closer investigation revealed that the $P$. griffithii in question had moderately heavy needle infection which, however, did not reach the stem before the needles were sloughed off. In the hybrids this inhibiting mechanism was broken down, the infection spread rapidly from the needles to the stem and heavy mortality of the seedlings was the result. The same was the case with hybrids of these $P$. griffithii with $P$. peuce. Thus a cross of two largely resistant white pine species yielded mostly susceptible hybrids through the action of complementary genes, in this case through the suppression of a (probably recessive) gene or genes, inhibiting the growth of the rust from the needles to the stem. If the inheritance of such an inhibiting mechanism is relatively simple (i.e. based on a few major genes) it should be possible to combine this with needle resistance found in $P$. peuce and some of the more resistant $P$. strobus materials and thus to create a double barrier to the rust. This should, in theory at least, reinforce the resistance already found in $P$. strobus and $P$. peuce. The breeding procedure could then follow a pattern already well established in the breeding for resistance in several agricultural and horticultural crops. We will then probably be able to obtain relatively rapid results and to introduce resistance into all the main climatic races of $P$. strobus 
and perhaps also into other white pine species. This will mean work on an interspecific level which has advantages of introducing heterosis, well known in hybrids of $P$. griffithii with $P$. strobus. We may, however, also introduce undesirable characteristics, such as poor stem form and excessive branchiness from $P$. griffithii or obtain these through the action of complementary genes found in the two species. Selection will then also have to be carried out in advanced generations to eliminate these undesirable effects. Another undesirable effect in work with major genes for disease resistance is the creation of an environment that is highly conducive to the origin and spread of more virulent mutations of the parasite in question. Such mutations can, within a very short time, cancel out much of the breeding work, as has been found repeatedly in work with resistance to stem rust in wheat.

Flor (4) has analyzed a rather convincing case of gene-for-gene mutation in flax and a rust that may well have applications elsewhere. One of the more promising measures against the survival of such mutations in the parasite is the simultaneous use of polygenes and major genes in breeding for resistance, and therefore all the present work, based on polygenic inheritance of resistance to blister rust may be of lasting value, even if major genes, making possible more rapid advances in breeding, will be discovered and used.

\section{Silvicultural Use of Resistant Materials}

Most forest trees are allogamous, i.e. usually cross-pollinating and probably contain many heterotic loci, which exhibit over-dominance when in a heterozygous condition. Hence positive heterosis may follow outbreeding and negative heterosis (or inbreeding depression) is the result of inbreeding.

As mentioned under diseases caused by facultative parasites, induction of heterosis is one of the methods in obtaining disease resistance. We have example of heterosis, following interracial and interspecific hybridization in poplars, larch, oak, birch, alder and most probably also in white pine. The effects of hybrid vigour can be retained by means of vegetative propagation, as in poplars. Hybrid vigour can also be maintained by careful selection of the parental clones used in seed orchards to produce hybrid seed of the desired combinations. Such clones can be maintained on any scale and for any length of time thus in this respect being similar to the pure lines used in producing hybrid corn and other plants. Resistance to disease may well be incorporated into such clones and included in tests for combining ability. The production of hybrid larch is a case in point and resistance to larch canker is one of the main assets of hybrid larch in comparison with ordinary European larch.

However, none of such hybrids are suitable for the establishment of naturally regenerating stands which will be necessary under most Canadian conditions. In this case, the F3 generation of an interracial or interspecific hybrid offers promise. The genetic breakdown usually experienced in the F2 of such hybrids is used for a very intensive selection of superior types, including screening for disease resistance, and the progenies are bulked to 
produce an F3 which itself, and in succeeding generations, is sufficiently heterogeneous for most forestry purposes. The one-shot method, as one may conveniently call the use of F1 hybrids only, is suitable for plantation forestry under intensive management and will, in all likelihood, be used as soon as suitable disease resistant materials of the most important forest tree species will become available.

The production of naturally regenerating tree materials with built-in resistance to certain diseases is the next step, designed to produce planting stock for the more extensive forest management practices in the less densely populated areas of northern Canada.

Most "improved" forest trees used today do not differ markedly from their wild ancestral forms and the rather difficult and slow process of transfer of polygenically inherited resistance from a wild species to a cultivar is as yet not necessary. Nor is it necessary for all individuals of an "improved" variety to be resistant to a certain disease, as long as the resistance is present in sufficient numbers of individuals at time of harvesting. Under such more extensive forms of management, it may become important to maintain a certain selection pressure for resistance to disease either by intensive screening of materials used for seed production or by maintaining the causal fungi in the tree population. Thus, in the case of blister rust in white pine, it might be advisable first to use heavily screened planting stock to establish seed orchards and then to maintain blister rust at a certain level in the plantations, to ensure resistance to it in the succeeding generations obtained by natural reproduction of such planted stands.

From all the above, it should be clear that breeding work on as wide a basis as possible is much preferable to work with materials adapted to a single geographic area only. It is, however, not possible to do this at a single research station. We cannot properly screen our northernmost provenances of white pine at Maple, Ont. nor can we profitably utilize disease resistance genes that may be available in Mexican white pine species nor in $P$. griffithii from low elevations. Just as in breeding for disease resistance in agricultural and horticultural crops, international co-operation, with free exchange of breeding materials, will become increasingly necessary.

\section{REFERENCES}

1. ALLARD, R. W. 1960. Principles of plant breeding. 485 pp. John Wiley \& Sons, New York \& London.

2. BINGHAM, R. T., SQUILLACE, A. E. and R. F. PATTON. 1956. Vigor, disease resistance, and feld performance in juvenile progenies of the hybrid Pimus monticola Dougl. $x$ Pinus strobus L. Zeitschrift für Forstgenetik und Forstpflanzenzüchtung 5(4): 104-112.

3. BINGHAM, R. T., SQUILLACE, A. E. and J. W. WRIGHT, 1960. Breeding blister-rust resistant western white pine II. First results of progeny tests including preliminary estimates of heritability and rate of improvement. Silvae Genetica $9(2): 33-41$,

4. FLOR, H. H. 1955. Host-parasite interaction in flax rust-its genetics and other implications. Phytopathology 45: 680-685.

5. MODE, C. J. 1958. A mathematical model for the co-evolution of obligate parasites and their hosts. Evolution 12(2): 158-165.

6. WHALEY, W. G. 1944. Heterosis. Botanical Review 10(8): 461-498. 